\title{
ANÁLISIS IDEOLÓGICO DE LAS POLÍTICAS PÚBLICAS EN EL DEPORTE DESDE UN ENFOQUE PSICOSOCIOLÓGICO EN LA CULTURA FÍSICA. EL CASO DE GUADALAJARA, JALISCO, EN LA REPÚBLICA MEXICANA
}

\author{
Pedro Reynaga-Estrada \\ Universidad de Guadalajara, Guadalajara, Jalisco, México
}

\begin{abstract}
Resumen
Estudio de revisión documental que gira en torno al análisis del discurso ideológico de las políticas públicas en el campo del deporte y los aspectos psicológicos implicados en la cultura física de dos documentos básicos que manifiestan la ideología dominante: La Ley Estatal del Deporte; orientada hacia el deporte competitivo, elitista y a la población juvenil; y la Vía RecreActiva de la zona Metropolitana de Guadalajara, que sus promotores pretenden darle un sentido idealista, ocultando los intereses socioeconómicos que la impulsaron.
\end{abstract}

Palabras clave: Psicología. Deporte. Cultura Física. Políticas Públicas.

\section{Introducción}

$\mathrm{L}$

os factores psicológicos están involucrados en toda actividad hucultura física de un pueblo implica asumir la responsabilidad de los gobiernos, de los grupos sociales y de los ciudadanos para mejorar el presente. Desde la psicología se pretenden aportar elementos que sean útiles en el diseño y construcción de las políticas públicas desde una visión socio histórica de la cultura física y las ciencias del deporte.

Aunque en sentido estricto el deporte está constituido por reglas o normas con el objetivo específico de mejorar el rendimiento para demostrarlo en la competencia, bajo condiciones de entrenamiento (ARIBAU, 1997; VARGAS, 1998) en este trabajo se entiende como categoría integradora referida a la actividad física o movimiento corporal humano en el que se experimenta placer durante su ejecución con sentido lúdico, de diversión, recreación y esparcimiento, sobre todo cuando se practica por distracción y con flexibilidad en las normas y la competencia (SILVA, REYNAGA \& QUIROGA, 2009; SIL- 
VA CAMARGO, 2002; PAULIN ZAMBRANO \& VARGAS, 2002). Esta definición es importante porque si se piensa en el deporte limitándolo a su carácter competitivo, se descuidarán las orientaciones recreativas, de actividad física o de tiempo libre en la mayoría de la población.

En este estudio se analizará la concepción que se tiene sobre el deporte, en el marco del concepto de cultura física, entendida, en su acepción más general de actividad humana, como el conjunto de productos materiales y simbólicos con relación a la acción motriz o movimiento humano (REYNAGA-ESTRADA, 2007). Debido a que el trabajo de la psicología aplicada a la cultura física es analizar las ideas, los valores, creencias y falsas conciencias manifestadas en los documentos y programas de actividad física y salud; este estudio analiza el significado psicosocial de las políticas públicas manifestadas en esos documentos. Como afirma Devís Devís, J. \& V. Pérez Samaniego (2001, p. 349): "se hace imprescindible que los profesionales identifiquen y tomen conciencia de la influencia social de estas distorsiones si la finalidad última de su actividad es mejorar la salud de la población". Las distorsiones o ideologías que se han construido colectivamente en las sociedades postindustriales alrededor de las representaciones simbólicas sobre la salud, la actividad física y el cuerpo humano... son sistemas de creencias, representaciones valores y prácticas sociales que conforman falsas o limitadas conciencias sobre la actividad física relacionada con la salud.

La intención del análisis del discurso, manifestado en documentos tales como leyes y documentos que expresan las políticas públicas hacia el deporte, es mostrar las falsas ideas que sólo son recursos de dominio y control político y psicosocial del grupo dominante. Las personas comúnmente siguen las modas, las creencias sociales dominantes y otras formas distorsionadas de ver y practicar la actividad física que chocan con la ética del profesional de la actividad física, por lo que dicho profesional debe cambiar estas ideas analizando los valores implícitos y explícitos de estas falsas ideologías (PEIRÓ VELERT \& DEVÍS DEVÍS, 2001).

La inclusión de políticas públicas que promueven el deporte no determina ni garantiza que cambie la disposición de los ciudadanos para que practiquen las actividades deportivas como un estilo de vida saludable. Y peor aún, cuando la población o algún sector social presentan resistencia al cambio pueden existir generar movimientos polí- 
ticos importantes que llevan a un gobierno a retractarse de estas tendencias. Ejemplo de estos abundan, pero sólo como muestra de estos frenos a las políticas públicas de apoyo a las actividades deportivas son la Ciclovía del gobierno de ciudad Juárez, Chihuahua en el 2004 por "pugnas políticas"; la incorporación de Tlaquepaque a la Vía RecreActiva por "resistencia de algunos comerciantes que no querían la Vía" (PÉREZ MARTÍNEZ, 2009, pp. 12 y 16) y el proyecto de Villa Panamericana en Guadalajara, Jalisco durante los años 2008, 2009 y posteriores. Este caso lo analizaremos más adelante en el apartado de la Vía RecreActiva en la Zona Metropolitana de Guadalajara.

La implementación de las políticas públicas para el deporte en la población, sea en el contacto persona a persona y/o en la comunicación masiva, la re significación de los valores de los participantes en un programa de actividad física puede ser una estrategia psico cultural que facilite el éxito. Parte de esta re significación es cautivar a los participantes para que la actividad física resulte una experiencia divertida, agradable y atractiva. Ello implica que el profesional evite que la práctica de la actividad física se convierta en un sacrificio o una experiencia negativa. "Si las personas disfrutan con la práctica de actividades será más fácil que su participación sea continuada en el futuro y la actividad adquirirá un nuevo significado en su vida" (PEIRÓ VELERT \& DEVÍS DEVÍS, 2001, pp. 330-331).

El objetivo del estudio fue analizar los principales documentos que expresan la ideología dominante de las políticas públicas en el Estado de Jalisco. Por ello se revisó un documento normativo general del deporte y uno específico y reciente del Gobierno del Estado sobre la vía RecreActiva. Resultando que la investigación cualitativa estudia los significados, experiencias, descritas verbalmente (COOLICAN, 2005), se analizó el discurso escrito de dos materiales que manifiestan las políticas públicas hacia el deporte del Gobierno del Estado de Jalisco: La Ley Estatal del Deporte y un documento generado por el Gobierno de Jalisco (2009) sobre la Vía RecreActiva. El análisis del discurso y la revisión documental sobre las concepciones de la educación física es un trabajo realizado en países como Brasil, por distintos investigadores como Mariante Neto, Favre, Myskiw \& Stigger (2010); Dias (2010) y muchos otros. En México es un trabajo exhaustivo realizado con profundidad por Eisenberg (2007) y sus colaboradores. 


\section{Jalisco y las políticas públicas en el deporte a nivel regional}

La expresión de la política pública también se concreta en las leyes y documentos normativos. En el estado de Jalisco, analizaremos los siguientes documentos: la Ley Estatal del Deporte, que es la norma jurídica que regula la política pública en el deporte; y un documento de promoción de la vía RecreActiva de Guadalajara.

Como ejemplo analizaremos el artículo primero de la Ley Estatal del Deporte:

Artículo $1^{\circ}$ - Las disposiciones de esta Ley son de orden público y de interés social, y tienen como finalidad establecer y fijar las bases para el funcionamiento del Sistema Estatal del Deporte, así como las normas de seguridad para la práctica del deporte, ello con el propósito de coadyuvar en la formación y desarrollo integral de los habitantes del Estado de Jalisco, en las actividades relacionadas con el deporte y la cultura física.

Como observamos, en primer lugar, la validez del estudio sobre las políticas públicas se marca en el primer renglón: cuando afirma que "las disposiciones de esta Ley son de orden público y de interés social".

Aunque su propósito es "coadyuvar en la formación y desarrollo integral de los habitantes del Estado de Jalisco", entra en contradicción cuando delimita su competencia institucional en la población juvenil, y en deporte, en el artículo tercero, que a la letra dice:

Artículo $3^{\circ}$ - La institución competente del Poder Ejecutivo del Estado de Jalisco en materia deportiva, será el Consejo Estatal para el Fomento Deportivo y el Apoyo a la Juventud...".

Ciertamente que en el artículo séptimo $\left(7^{\circ}\right)$ incluye a la educación básica (población infantil) para que obtengan 5 horas de ejercicio corporal mediante un programa diseñado y ejecutado por el CODE y la Secretaría de Educación Jalisco "que coadyuven en la formación y desarrollo integral del educando".

Igualmente solamente en dos fracciones (fracción $\mathrm{X}$ y fracción $\mathrm{V}$ de los artículos noveno y décimo tercero, respectivamente) se refiere a la población de la tercera edad, personas con discapacidades y demás poblaciones especiales. De allí en más no hace referencia a otra población que no sea la juvenil.

La tercera parte en el análisis del primer artículo (y en toda la Ley, como observamos en los artículos previos) es la limitación de su concepción que está dirigida al deporte competitivo, por lo que las activi- 
dades físicas, recreativas, de tiempo libre, y otras, escapan a su atención y observancia jurídica- política; aunque al final del artículo primero menciona a las "actividades relacionadas con el deporte y la cultura física". El incluir al final del párrafo la "cultura física" es solamente una excepción en toda la ley. No la vuelve a incluir en todo el documento. Por ello, en el discurso y en la práctica, la atención exclusiva al deporte competitivo muestra una exclusión de otras formas de ejercicio no competitivo, reflejando ésa realidad cotidiana de exclusión de las personas. Como en el pasado, "los primeros gestos de exclusión los cometemos los maestros en el momento de elegir a los mejores jugando contra los no tan destacados, mientras el resto cumple la función de precoces espectadores" (AGUILAR, 2009, p. 93). Las políticas públicas hacia el deporte han fallado porque sólo se convierte en un fenómeno de espectáculo, en donde la gran mayoría solamente observa. Y los datos son reveladores: Sólo el 5\% de la población mundial practica formalmente el deporte (GARI \& YANZA-MERA, 2009).

La orientación de la política pública en la Ley Estatal del Deporte está definida, como su nombre lo dice, al Deporte. Y la concepción que tiene sobre este fenómeno es en el sentido de la competencia, excluyendo las actividades recreativas, lúdicas y de tiempo libre, según se puede observar a lo largo del documento.

\section{La Via RecreActiva en la zona metropolitana de Guadalajara}

La Zona Metropolitana de Guadalajara tiene aproximadamente cinco millones de habitantes. La Vía RecreActiva de la Zona Metropolitana de Guadalajara "se extiende por 65 kilómetros en el territorio de los cinco municipios" (Guadalajara, Zapopan, Tlaquepaque, Tonalá y Tlajomulco). "Dura 6 horas cada domingo y promedia 253 mil visitantes por jornada: 205 mil en bicicleta, 12 mil en patines o patinetas (...)" (PÉREZ- MARTÍNEZ, 2009b, p. 123). Há sido visitada 32 millones de veces desde septiembre del año 2004 en que inició, con lo que lleva 262 domingos realizados hasta finales de 2009. El modelo fue inspirado en la Ciclovia dominical de Bogotá, Colombia de 120 kilómetros de largo y PÉREZ- MARTÍNEZ, 2009a).

Con estas cifras ha sido considerada como un proyecto exitoso por el gobierno del Estado de Jalisco que directamente la promovió; y 
particularmente por el gobernador del Estado: Emilio González Márquez (PÉREZ- MARTÍNEZ, 2009a).

Un elemento psico- socio- educativo es el análisis de la ideología, falsa conciencia o valores sobre el deporte que se puede hacer como recurso para favorecer el cambio de actitudes y conducta hacia la práctica de las actividades deportivas y en este caso recreativas, contrarrestando la tendencia hacia el sedentarismo del mundo actual. Una ventaja de la Vía RecreActiva es que sensibiliza poco a poco a la población para participar en actividades deportivas (recreativas) como una oportunidad de esparcimiento y uso del tiempo libre, a diferencia de la Ley Estatal del Deporte, que solamente se centra en el deporte competitivo.

Pero de allí a como se pretende exponer en el libro que se difunde por el Gobierno de Jalisco (2009), hay una enorme distancia. Por ello, es interesante sacar a luz algunos valores velados que se promueven. Por ejemplo, el corte idealista que pregona, casi de cuento; no solamente como un recurso literario y de redacción, sino como un recurso ideológico. Expresiones como "cuenta la leyenda"... (PÉREZ- MARTÍNEZ, 2009a, p. 13) o "La via recreactiva nos regresó al vecino, con nombre y rostro; al barrio de enfrente, con sus fiestas y sus servicios; la calle volvió a ser nuestra y el espacio público el lugar común"... “(...) cada domingo 250 mil tapatíos se reconocen los unos a los otros: el cholo, la monja, el empresario, el bolero, el estudiante, el niño, el plomero, el fresa, el mocho, el liberal, el nuevo tapatío, el de abolengo (...)" (PETERSEN- FARAH, 2009, p. 63). Como si con seis horas que dura la Ciclovía se lograra humanizar a los ciudadanos que pasan 166 horas deshumanizados siguiendo esta lógica (de los 7 días a la semana menos las 6 horas) y como si con estas seis horas en este espacio se diluyeran también las clases sociales.

El deporte y la recreación, impregnados de los valores del sistema capitalista no escapan a la lógica de compra- venta. Señala Castellani (2009) que en las sociedades y naciones todo se vende y se compra: la educación, la salud, el agua, el deporte, con lo que se convierten en grandes centros comerciales. El supuesto "tiempo libre" también se esclaviza bajo ésta lógica, subordinado a la reproducción del mismo, se convierte en un elemento de consumo. La configuración del espacio y el tiempo de las personas consolidan los intereses y valores propios de la sociedad de consumo y a la industria cultural del entretenimiento. 
Cuando los pueblos se convierten en ciudades son atractivas para los grandes socios comerciales, convierten grandes plazas de consumo y los ciudadanos ocupan su tiempo libre en las compras, constituyendo un estilo de vida que disminuye la posibilidad de ocupar el tiempo libre en actividades deportivas.

La iniciativa de la Vía Recreactiva de Guadalajara, nació y prosperó no solamente por buenas intensiones y deseos de beneficios desinteresados, sino por intereses económicos: "cuando un grupo de joyeros decidió rescatar la plaza tapatía (...) cuando descubrieron que era mucho más seguro trabajar en centros joyeros... [Empezaron] a involucrarse en los temas de desarrollo de ciudad y los espacios públicos [y] también querían buscar solución a los problemas viales del centro histórico...Respaldados por el Consejo de Cámaras Industriales de Jalisco [se hicieron las gestiones con empresarios, gobierno e instituciones [como por ejemplo] ciudades públicas"... y se encontró el apoyo del gobernador del Estado y los presidentes municipales de la Zona Metropolitana (PÉREZ- MARTÍNEZ, 2009a, pp. 10 y ss).

El deporte está ligado a los aspectos socioeconómicos. La sociedad capitalista actual está empezando una grave crisis que por esencia se originó en sus contradicciones internas desde sus inicios. Trigo, Inacio \& Montoya (2009) señalan que el capitalismo enseña cómo acumular, cómo obtener ganancia, cómo no utilizar los bienes, cómo no utilizar la riqueza. El apetito voraz del capitalismo y su necesidad desmedida de consumo ha llevado al mundo al borde del agotamiento de los recursos naturales (y humanos). El atesoramiento y la acumulación de recursos que no se necesitan convierte a los servicios en inservibles, los bienes en males; porque quienes necesitan esos recursos no los pueden utilizar. Los artículos perecederos perecen, no se consumen; mientras que la gran mayoría de la población no tiene acceso a ellos, aunque allí estén, acumulados, porque quien no tiene el dinero para comprarlos no puede consumirlos. Eso mismo pasa en algunas ciudades con el deporte; aunque existan los espacios deportivos, los gimnasios, etc. si no se tiene el dinero para pagar el ingreso, la membrecía, el pago de la mensualidad o anualidad, no se puede acceder a él. Y hay quienes tienen dinero para pagarlo, pero no lo hacen, lo acumulan y no practican el deporte. La riqueza se acumula en algunos pocos y la pobreza se distribuye en muchos. Se forza la producción y se convierte en "basura", que no es sino un recurso no utilizado y que en el caso de los productos perecederos al transportarlos a un medio que no es 
donde deben de estar en su estado natural, contaminan, dañan la naturaleza, pero sobretodo (y como parte de ella) a otros seres humanos. No sólo es triste y doloroso el daño ocasionado a los niños, jóvenes y adultos que mueren de hambre y enfermedades, sino también por los que tienen una vida constante de carencias. Y parte de estas carencias es la imposibilidad de practicar el deporte. Tenemos algunos grandes o hermosos espacios deportivos que están olvidados, desperdiciados o aprovechados mínimamente, sólo reservados para quienes pueden pagar el ingreso.

Pero tampoco podemos pensar en brindar la oportunidad de la práctica deportiva a la población, sino se le puede ofrecer al menos la posibilidad de empleo, remuneración, alimentación y educación.

La ventaja de la Via RecreActiva es que se ofrece como un espacio libre y gratuito para todas las personas sin distinción de clase social, religión o nível educativo (sin querer decir que se eliminan o diluyencomo pretenden hacer creer en el texto-; solo se ven y si bien va, conviven).

La desventaja es que por más amplia que sea la ruta y por más que avance que tenga está limitada espacio- temporalmente. Si queremos un espacio democrático para la práctica recreativa como parte del deporte debemos expandirla a todas las calles todos los dias para la sana convivencia de todas las personas y todos los actores sociales que se incluyen en la movilidad urbana: automovilistas, motociclistas, ciclistas, peatones y personas con capacidades diferentes.

Para ello, se debe asumir el mismo apoyo decidido del gobierno del Estado para reglamentar el respeto entre los actores de movilidad mencionados, máxime ante un transporte público deshumanizado y con conductores con gran cantidad de estrés citadino.

Otra desventaja es que al afectar intereses o necesidades de libre tránsito de los automovilistas (acostumbrados a la prioridad que se les da en los espacios urbanos) asumen con molestia una política pública como la Via Recreactiva por que es producto de una imposición y del interes del gobierno en turno.

\section{Consideraciones finales}

Gracias al análisis del discurso de las políticas públicas en el deporte se pueden descubrir las contradicciones entre las intenciones formuladas y los fundamentos y orientaciones que se exponen en los 
documentos estudiados, para develarlos y proponer la redirección de los mismos. Realizando este tipo de estudios se deben impulsar y sensibilizar a los gobernantes para que se promuevan políticas públicas democráticas en el deporte, en que se incluyan a todas las personas y grupos sociales que puedan y deban practicarlo para integrar coherentemente las intenciones con las orientaciones de documentos tan importantes como la Ley Estatal del Deporte. Con ello se puede garantizar que las actividades físicas y deportivas sean un derecho de acceso libre y gratuito al menos en todas las dependencias de gobierno.

Las políticas públicas en el deporte, deben instrumentar la forma en que se incluya a los jóvenes, personas mayores, niños y a todo ciudadano que pueda realizarlo, porque realizado en forma adecuada es benéfico para todas las edades.

Debemos pensar cómo impulsar las actividades deportivas, cómo vencer los obstáculos para lograr que las personas puedan practicar la actividad física. La educación física es un buen sistema para iniciar a favorecer el cambio de valores, actitudes y conductas hacia el deporte, pero se debe cuidar que no siga la orientación tradicional hacia la competición. Además la educación física debe extenderse hacia los campos menos formales del sistema educativo para incidir no sólo en la población infantil y juvenil; sino en la población que no ha tenido acceso a la educación: la población adulta y adulta mayor. El deporte es solamente una actividad, una herramienta que se puede utilizar para mejorar la salud y la educación humanas.

Ideological analysis of public policy in the sport with psychosociological vision on physical culture. Guadalajara case, Jalisco, México Republic

\begin{abstract}
Study based on literature review around the analysis of ideological discourse of public policy in the sport's field and the psychological aspects related to physical culture of two basic documents that describes the dominant ideology: State Law Sport oriented to competitive sport, elite sport and the youth population; and the "Via Recreactiva" in metropolitan area of Guadalajara city, which its supporters pretend to make a sense of idealism, hiding the socioeconomic interest that made possible its creation.
\end{abstract}

Keywords: Psychology. Sport. Physical Culture. Public Policies. 
Análise ideológica das políticas públicas no esporte com enfoque psicossociológico na cultura física. $O$ caso de Guadalajara, Jalisco, México

\section{Resumo}

O presente texto, construído a partir de pesquisa documental, analisa o discurso ideológico a respeito das políticas públicas no esporte e dos fatores psicológicos implícitos na cultura física de dois documentos que manifestam a ideologia dominante: a Lei Nacional do Esporte, que se orienta para o esporte competitivo, elitista e para a população juvenil; e a Via RecreAtiva da região metropolitana de Guadalajara, cujos promotores buscam conferir-lhe um sentido idealista, ocultando os interesses socioeconộmicos que a impulsionaram.

Palavras-chave: Psicologia. Cultura Física. Esporte. Políticas Públicas.

\section{Referências}

AGUILAR EGÜES, M. El largo camino de la democratización en el deporte. En: TAVOSNANSKA, P. H. (Ed.). Democratización del Deporte, la educación física y la recreación. Aportes a la Integración Regional y la Cooperación Internacional. Buenos Aires: Editorial Biotecnológica S.R.L., 2009. p. 92-96.

ALLMER, H. Actividad deportiva y abstención del deporte entre las personas mayores. En: DENK, H. (Ed.). Deporte para mayores. Barcelona: Paidotribo, 2003. p. 10-23.

AMAYA, G. D. \& BARBERO, C. A. Hacia una política del deporte. En: TAVOSNANSKA, P. H. (Ed.). Democratización del Deporte, la educación física y la recreación. Aportes a la Integración Regional y la Cooperación Internacional. Buenos Aires: Editorial Biotecnológica S.R.L., 2009. p. 159-169.

AQUINO LÓPEZ, H. E. (Ed.). Práctica docente de la educación física en la escuela primaria. Zapopan, México: Instituto Superior de Investigación y Docencia para el Magisterio, 2008.

AQUINO LÓPEZ, H. E., OROZCO-LÓPEZ, M. T. \& FIGUEROADEL-REAL, M. I. La política educativa que regula la formación y la práctica docente de educación física. En: AQUINO LÓPEZ, H. E. (Ed.). Práctica docente de la educación física en la escuela Primaria. 1. ed. Zapopan, México: Instituto Superior de Investigación y Docencia para el Magisterio, 2008. p. 47-80. 
ARIBAU C. Diccionarios.com. Barcelona, España. 3ra edición. http://diccionarios.com. ,1997.

BECKER, B. El cuerpo y su implicancia en el área emocional. Lecturas de Educación Física y Deportes. Revista Digital. Disponible en: http://www.sportquest.com/revista/efd13/bennoe.htm [2000, 29 de febrero], 1999.

BROHM, J. M. Sociología Política del Deporte. México: Fondo de Cultura Económica, 1982.

CASTELLANI FILO, L. Las políticas de deporte y tiempo libre en el contexto de la integración latinoamericana. En: TAVOSNANSKA, P. H. (Ed.). Democratización del Deporte, la educación física y la recreación. Aportes a la Integración Regional y la Cooperación Internacional. Buenos Aires: Editorial Biotecnológica S.R.L., 2009. p. 149158.

COOLICAN, H. Métodos de investigación y estadística en psicología. 3. ed. México: El Manual Moderno, 2005.

DCAK, J. eporte al alcance de todos. En: TAVOSNANSKA, P. H. (Ed.). Democratización del Deporte, la educación física y la recreación. Aportes a la Integración Regional y la Cooperación Internacional. Buenos Aires: Editorial Biotecnológica S.R.L., 2009. p. 85-91.

DEVÍS DEVÍS, J. \& PÉREZ SAMANIEGO, V. La ética en la promoción de la actividad física relacionada con la salud. En: DEVIS DEVÍS, J. (Ed.). La educación física, el deporte y la salud en el siglo XXI. España: Editorial Marfil, 2001. p. 341- 355.

DIAS, C. Novos sonhos de verão sem fim: surfe, mulheres e outros modos de representação. Revista Brasileira de Ciências do Esporte, Campinas, 32(2-4), p. 75-78,

2010.

DIRECCIÓN GENERAL DE EDUCACIÓN FÍSICA. Programa de Educación Física. México, D. F.: Secretaría de Educación Física, 1994. 
EISENBERG WIEDER, R. (Ed.). Corporeidad, movimiento y educación física. 1. ed. México, D.F: Consejo Mexicano de Investigación Educativa, 2007. (Estudios conceptuales, v. 1).

EISENBERG WIEDER, R. Antecedentes. En: EISENBERG WIEDER, R. (Ed.). Corporeidad, movimiento y educación física. 1. ed. México, D.F: Consejo Mexicano de Investigación Educativa, 2007. p. 23-41. (Estudios conceptuales, v. 1).

EISENBERG WIEDER, R. \& GRASSO, A. Introducción conceptual a corporeidad, movimiento y educación física. En: EISENBERG WIEDER, R. (Ed.). Corporeidad, movimiento y educación física. 1. ed. México, D.F: Consejo Mexicano de Investigación Educativa, 2007. p. 43-83. (Estudios conceptuales, v. 1).

GARI, G. \& YANZA MERA, P. A. Ejercicio democrático en el deporte, la actividad física y la recreación: utopía o realidad posible. En: TAVOSNANSKA, P. H. (Ed.). Democratización del Deporte, la educación física y la recreación. Aportes a la Integración Regional y la Cooperación Internacional. Buenos Aires: Editorial Biotecnológica S.R.L., 2009. p. 27-37.

GOBIERNODE JALISCO. Una vía para vivir la ciudad. 1. ed. México, D.F.: Andrés Bello Editorial de C.V. Printing Arts México, S de R.L./ C.V., 2009.

LEY ESTATAL DEL DEPORTE PARA EL ESTADO LIBRE Y SOBERANO DE JALISCO, 18008 (1999).

LEYE, M. Deporte y tensiones psíquicas a edades avanzadas. En: DENK, H. (Ed.). Deporte para mayores. Barcelona: Paidotribo, 2003. p. 69-84.

MARIANTE NETO, F. P., FAVRE MIRANDA, C., MYSKIW, M. \& STIGGER, M. P. Muhamad Ali, um outsider na sociedade Americana? Revista Brasileira de Ciencias do Esporte, 32(2-4), p. 105-122, 2010 .

MARTÍNEZ MOYA, A. El árbol desdibuja su vuelo: los maestros y la política educativa en Guadalajara 1990-2000. Cuadernos de Investigación. Guadalajara, México: Instituto Superior de Investigación y Docencia para el Magisterio (ISIDM), 2005. p. 71-98. 
PAULIN ZAMBRANO, L. \& VRGAS, R. Diccionario básico de conceptos sobre actividades físico- deportivas y recreativas. México, D.F.: Ediciones Supernova, 2002.

PAZ SANCHEZ, B. \& DURÁN-ARCE, J. (1987). El problema de la cultura física: una reflexión necesaria. Cultura Física. Revista Científico-Metodológica del ISCF Manuel

Fajardo (1), p. 73-84.

PEIRÓ VELERT, C. \& DEVÍS-DEVÍS, J. La escuela y la comunidad: principios y propuestas de promoción de la actividad física relacionada con la salud. En: DEVÍS DEVÍS, J. (Ed.). La educación física, el deporte y la salud en el siglo XXI. 1. ed. España: Editorial Marfil, 2001. p. 323-339.

PÉREZ MARTÍNEZ, A. (2009a). La historia de una carambola. La gestión de la Vía RecreActiva. En: GOBIERNO DE JALISCO (Ed.). Una vía para vivir la ciudad. 1. ed. México, D.F.: Andrés Bello Editorial de C.V. Printing Arts México, S de R.L./C.V., 2009. p. 1017.

PÉREZ MARTÍNEZ, A. (2009b). Las manos que cultivan la Vía. En: GOBIERNO DE JALISCO (Ed.). Una vía para vivir la ciudad. 1. ed. México, D.F.: Andrés Bello Editorial de C.V. Printing Arts México, S de R.L./ C.V., 2009. p. 122- 129.

PETERSEN FARAH, D. Y la calle fuimos nosotros. En: GOBIERNO DE JALISCO (Ed.). Una vía para vivir la ciudad. 1. ed. México, D.F.: Andrés Bello Editorial de C.V. Printing Arts México, S de R.L./ C.V., 2009. p. 60-64.

PUGGA, A. \& MOLINA-BEDOYA, V. A. Capítulo IV. Legislación en deportes, educación física y recreación. En: TAVOSNANSKA, P. H. (Ed.). Democratización del Deporte, la educación física y la recreación. Aportes a la Integración Regional y la Cooperación Internacional. 1. ed. Buenos Aires: Editorial Biotecnológica S.R.L., 2009. p. 39-51.

PÜHSE, U. La experiencia del deporte y el ejercicio en la tercera edad. En: DENK, H. (Ed.). Deporte para mayores. Barcelona: Paidotribo, 2003. p. 25- 42. 
RAMÍREZ SNCHEZ SANZ. Encarta enciclopedia. España: Microsoft, 2000.

REYNAGA ESTRADA, P., LÓPEZ-TAYLOR, J. R., GONZÁLEZVILLALOBOS, M. F. \& MIRANDA-CAMPOS, A. M. El debate necesario entre la educación física, la educación física y la actividad física: una reflexión para la formación profesional del estudiante. Revista de Educación y Desarrollo (7), p. 27-34, 2007.

SÁNCHEZ CEREZO, SERGIO. Diccionario de las Ciencias de la Educación. Madrid: Santillana, 1995.

SCHUBERT, A. \& BÖS, K. Sobre la capacidad del rendimiento motor de las personas mayores. En: DENK, H. (Ed.). Deporte para mayores. 1. ed. Barcelona: Paidotribo, 2003. p. 175-198.

SILVA, A. M., REYNAGA-ESTRADA, P. \& QUIROGA, S. R. Ciencia, educación física, deporte y recreación. Aproximaciones epistemológicas y sociales desde el sur. En: TAVOSNANSKA, P. H. (Ed.). Democratización del Deporte, la educación física y la recreación. Aportes a la Integración Regional y la Cooperación Internacional. Buenos Aires: Editorial Biotecnológica S.R.L., 2009. p. 47-57.

SILVA-CAMARGO, G. Diccionario básico del deporte y la educación física. 3. ed. Armenia, Colombia: Editorial Kinesis, 2002.

TRIGO, E., TORO, S., INACIO, H. L. \& MONTOYA, H. Ecología y vida. Conexiones con la educación física. En: TAVOSNANSKA, P. H. (Ed.). Democratización del Deporte, la educación física y la recreación. Aportes a la Integración Regional y la Cooperación Internacional. Buenos Aires: Editorial Biotecnológica S.R.L., 2009. p. 53-62.

VARGAS, RENÉ. Teoría del entrenamiento. Diccionario de conceptos. Dirección General de Actividades Deportivas y Recreativas. México: UNAM, 1998.

Recebido em: 27/11/2011

Revisado em: 18/03/2012

Aprovado em: 03/05/2012

Pensar a Prática, Goiânia, v. 15, n. 4, p. 821-1113, out./dez. 2012 1106 


\section{Endereço para correspondência}

preynagaestrada@yahoo.com.mx.

Pedro Reynaga-Estrada

Universidad de Guadalajara

Circuito Hacienda del Carmen Oriente, 25

Col. Hacienda Real, 2a . C.P.

Sección, Tonalá Jalisco.

45428 - México. 